\title{
A SITUAÇÃO DE TRABALHO DO PESSOAL DE ENFERMAGEM NO CONTEXTO DE UM HOSPITAL ARGENTINO: UM ESTUDO SOB A ÓTICA DA ERGONOMIA*
}

Azucena Del Valle Royas**

Maria Helena Palucci Marziale***

ROYAS, A.D.V.; MARZIALE, M.H.P. A situação de trabalho do pessoal de enfermagem no contexto de um hospital argentino: um estudo sob a ótica da ergonomia. Rev.latino-am.enfermagem, Ribeirão Preto, v. 9, n. 1, p. 102108, janeiro 2001.

Com o objetivo de caracterizar a situação de trabalho do pessoal de enfermagem num hospital Argentino através da identificação das características dos trabalhadores, das atividades executadas e do ambiente laboral, utilizou-se como pressuposto metodológico a Ergonomia. Buscando o diagnóstico da situação de trabalho foram realizadas entrevistas com 74 trabalhadores, sendo 17 enfermeiros e 57 auxiliares de enfermagem. Por meio dos resultados obtidos, constatou-se que a situação de trabalho estudada apresenta problemas relacionados a organização do trabalho, divisão de tarefas, falta de política voltada à melhoria da capacitação profissional e a segurança no trabalho, promoção à saúde dos trabalhadores, adequação de materiais $e$ equipamentos. Com base da Ergonomia de correção são apresentadas sugestões.

UNITERMOS: enfermagem, trabalho, ergonomia

\section{INTRODUÇÃO}

$\boldsymbol{A}_{\text {s }}$ condições de trabalho oferecidas pelos hospitais, as peculiaridades das tarefas de enfermagem, a crise econômica advinda da globalização, as dificuldades do setor saúde, a carência de recursos humanos e materiais e a constante preocupação com o processo de atualização objetivando acompanhar os avanços técnicos-científicos são fatores que contextualizam a situação de trabalho do pessoal de enfermagem em vários países.

Segundo AGUDELO (1995), o trabalho de enfermagem caracteriza-se por uma série de atividades e tarefas descontínuas, envolve múltiplos graus de responsabilidade e complexidade segundo a relação e o tipo de função exercida, acrescido da carga advinda do convívio com a morte, a dor e o sofrimento humano.

Essa situação induz a necessidade de um contínuo processo de adaptação entre trabalho e trabalhador, para que a assistência prestada ao cliente seja de boa qualidade e o trabalhador desempenhe suas atividades sem prejuízo de sua saúde física e mental.

A presente realidade fundamentou a pesquisa ora apresentada, a qual, através da utilização da abordagem ergonômica procurou analisar a situação de trabalho e saúde dos trabalhadores de enfermagem de um hospital regional da Argentina, onde os estudos relativos a essa temática são incipientes exigindo investimentos com o propósito de melhorar as condições de trabalho.

A seguir apresenta-se o referencial teórico utilizado, contextualizando os aspectos considerados essenciais para o entendimento da situação de trabalho do pessoal de enfermagem.

O hospital é considerado um ambiente de risco por abrigar uma série de agentes que podem ser nocivos quando não controlados. Dentre os fatores de riscos do trabalho da enfermagem nos hospitais evidenciam-se: riscos físicos (inadequação de iluminação, temperatura e ruídos); riscos químicos (medicamentos, desinfetantes esterilizantes e gases anestésicos); riscos biológicos (vírus bactérias, fungos); risco psíquico (excesso de trabalho, relacionamento humano difícil); risco social (agressões físicas e/ou verbais) e riscos ergonômicos (esquema de trabalho em turnos, carga física e mental, mobiliários inadequados).

A Organização Internacional de Trabalho (OIT)

\footnotetext{
* Artigo extraído de Dissertação de mestrado apresentada a Escola de Enfermagem de Ribeirão Preto da Universidade de São Paulo

** Mestranda do Programa de Pós-Graduação da Escuela de Enfermería de la Universidad Nacional del Patagonia Austral

*** Professor Doutor do Departamento de Enfermagem Geral e Especializada da Escola de Enfermagem de Ribeirão Preto da Universidade de São Paulo. Endereço: Av. Bandeirantes, 3900 - Monte Alegre - 14040-902 - Ribeirão Preto - São Paulo - Brasil. E-mail: marziale@glete.eerp.usp.br
} 
em 1985, através da Recomendação $\mathrm{n}^{0} 171$, propõe a preservação da saúde dos trabalhadores nos locais de trabalho mediantes ações de melhorias às condições do trabalho, inclusive para o pessoal que trabalha em hospital (SANZ-GALLÉN et al., 1995). Muitos países ratificaram a referida recomendação da OIT dentre eles a Argentina.

A Lei 19.587 (ARGENTINA, 1993), regulamenta as técnicas de higiene e segurança no trabalho contemplando as medidas sanitárias e prevencionistas, de tutela ou de qualquer outra índole que tenham por objeto: proteger a vida, preservar e manter a integridade psicofísica dos trabalhadores; prevenir, reduzir, eliminar ou afastar os riscos dos distintos postos de trabalho; estimular e desenvolver atitude positiva a respeito da prevenção dos acidentes ou enfermidades que possam derivar-se da atividade laboral.

Assim torna-se, também legalmente, necessário buscar a melhoria das condições de trabalho nos hospitais preservando a saúde dos trabalhadores que atuam nessas instituições e melhorando a qualidade da assistência prestada ao paciente.

Diante dos vários fatores interferentes na relação saúde-trabalho-doença, uma série de abordagens metodológicas tem sido utilizadas para diagnosticar e intervir nas condições de trabalho, dentre elas a abordagem ergonômica a qual foi selecionada para nortear esta investigação.

IIDA (1997, p. 1) menciona uma definição concisa da ergonomia elaborada pela Ergonomics Research Society, Ergonomia é o estudo do relacionamento entre o homem e o seu trabalho, equipamento e ambiente, e particularmente a aplicação dos conhecimentos de anatomia, fisiologia e psicologia na solução dos problemas surgidos desse relacionamento.

O objetivo da Ergonomia é assegurar satisfação, segurança e bem-estar dos trabalhadores em relação ao trabalho, tarefa, equipamento, ao meio ambiente e ao comportamento entre trabalhadores.

A intervenção ergonômica na situação de trabalho pode ser realizada em diferentes fases do processo de trabalho, tanto na organização laboral (condições laborais e atividades de trabalho) como no meio ambiente de trabalho.

Os componentes analisados na situação de trabalho na abordagem ergonômica são: o homem (trabalhador), a atividade e o ambiente de trabalho. As características individuais tais como idade, sexo, estatura, peso, compleição física, capacidade visual e auditiva, habilidade motora, personalidade, resistência à fadiga, diferenças de formação profissional dentre outras, podem interferir na forma do indivíduo conviver no trabalho.

O princípio geral da Ergonomia diz que as máquinas e o ambiente de trabalho devem ser adaptados ao homem e devido as grandes diferenças individuais existentes entre as pessoas, elas podem ser adequadamente selecionadas, possibilitando modificações pelo treinamento (IIDA,1997).

A atividade de trabalho, segundo o referido autor é composta pela tarefa prescrita (formal), geralmente definidas pela gerência, para atender aos objetivos fixados pela instituição e tarefa real a atividade efetivamente realizada pelo trabalhador, face às regras de funcionamento da instituição, ao contexto de realização do trabalho e à saúde e eficiência que os trabalhadores chegam a desempenhar, obedecendo a um processo evolutivo.

Muitas vezes as tarefas prescritas se distanciam das efetivamente realizadas devido as relações estabelecidas entre o homem e o trabalho, a filosofia da instituição e dos recursos humanos e materiais disponíveis.

O ambiente de trabalho é um conjunto de fatores interdependentes, que atua direta e indiretamente na qualidade de vida das pessoas e nos resultados do próprio trabalho. A visão global das influências do trabalho facilita a compreensão das dificuldades e desconfortos, da insatisfação, dos baixos desempenhos, das doenças camufladas e/ou na ocorrência de acidentes e incidentes do trabalho (FISCHER \& PARAGUAY, 1989).

Segundo IIDA (1997) o ambiente de trabalho também pode expressar grande fonte de tensão no trabalho; ambiente desfavorável com excesso de calor, ruídos, vibrações, causa desconforto, aumenta os riscos de acidentes e podem provocar danos consideráveis à saúde (enfermidades laborais e acidentes de trabalho).

\section{OBJETIVOS}

\section{Objetivo geral}

- Caracterizar a situação de trabalho do pessoal de enfermagem de um hospital regional Argentino.

\section{Objetivos específicos}

- Identificar as características dos trabalhadores de enfermagem;

- Identificar as atividades laborais executadas;

- Identificar as características do ambiente de trabalho.

\section{MATERIAL E MÉTODO}

Local: Hospital Regional da Argentina (HR). Trata-se de um hospital público, de 216 leitos, presta assistência a nível de internação, ambulatório, domicilio e de urgência e em caso de catástrofe.

População: Composta por 169 trabalhadores de 
enfermagem, pertencentes às categorias de Enfermeiros e Auxiliares de Enfermagem.

Amostra e critérios de inclusão: A amostra constituiuse de 74 sujeitos pertencentes as categorias Enfermeiros universitários $* * * *$; Enfermeiros não universitários $* * * * *$ e Auxiliares de Enfermagem******. A seleção dos sujeitos deu-se por amostra probabilística aleatória simples, sendo utilizados os seguintes critérios: aceitar participar voluntariamente da pesquisa e ter disponibilidade para agendamento da entrevista.

Coleta de dados: Foram realizadas entrevistas estruturadas com utilização de um protocolo de coleta de dados contendo perguntas abertas e fechadas. Abordando o referido instrumento foi apreciado por três pesquisadoras em relação ao conteúdo, clareza e objetividade sendo considerado adequado.

Análise dos dados: As respostas foram agrupadas e categorizadas a fim da elaboração de um banco de dados para posterior análise através do programa de computador Epi Info 6, versão 6.04b. Os dados foram apresentados de forma descritiva com distribuição numérica e percentual simples e com cruzamento de alguns itens investigados.

Aspectos éticos: O projeto foi aprovado pelo Comitê de Capacitação e Docência do hospital em estudo e foram seguidas as normas da Resolução 196/96 do Ministério da Saúde referente às normas éticas de pesquisas envolvendo seres humanos (BRASIL. MS., 1997).

\section{RESULTADO E DISCUSSÃO}

A exposição dos resultados encontre-se descrita segundo os três componentes da situação de trabalho considerados nesta investigação: o homem, a atividade e o ambiente.

\section{Características do homem (trabalhador de enfermagem)}

Através da Tabela 1 são apresentados alguns dados sobre as características dos trabalhadores de enfermagem da amostra estudada.

Tabela 1 - Número e porcentagem de trabalhadores de Enfermagem de um H.R., segundo ao sexo, estado civil e idade em anos e a categoria profissional. Argentina, 1999

\begin{tabular}{|c|c|c|c|c|c|c|c|c|}
\hline \multirow{3}{*}{ DADOS PESSOAIS } & \multicolumn{8}{|c|}{ CATEGORIA PROFISSIONAL } \\
\hline & \multicolumn{2}{|c|}{$\begin{array}{l}\text { Enfermeiro } \\
\text { Universitário }\end{array}$} & \multicolumn{2}{|c|}{$\begin{array}{l}\text { Enfermeiro não } \\
\text { Universitánio }\end{array}$} & \multicolumn{2}{|c|}{ Auxiliar de Enfermagem } & \multicolumn{2}{|c|}{ TOTAL } \\
\hline & $n^{0}$ & $\%$ & $n^{0}$ & $\%$ & $n^{0}$ & $\%$ & $n^{0}$ & $\%$ \\
\hline \multicolumn{9}{|l|}{ Sexo: } \\
\hline Feminino & 10 & 13,5 & 3 & 4,1 & 42 & 56,7 & 55 & 74,3 \\
\hline Masculino & 2 & 2,7 & 2 & 2,7 & 15 & 20,3 & 19 & 25,7 \\
\hline Total & 12 & 16,2 & 5 & 6,8 & 57 & 77,0 & 74 & 100,0 \\
\hline \multicolumn{9}{|l|}{ Estado civil: } \\
\hline Solteiro & 5 & 6,8 & - & - & 11 & 14,8 & 16 & 21,6 \\
\hline Casado/A masiado & 6 & 8,1 & 3 & 4,1 & 35 & 47,3 & 44 & 59,5 \\
\hline Viúvo & - & - & - & - & 2 & 2,7 & 2 & 2,7 \\
\hline Separado & 1 & 1,4 & 2 & 2,7 & 9 & 12,2 & 12 & 16,2 \\
\hline Total & 12 & 16,2 & 5 & 6,8 & 57 & 77,0 & 74 & 100,0 \\
\hline \multicolumn{9}{|l|}{ Idade: } \\
\hline 21 |--- 30 & 4 & 5,4 & 3 & 4,1 & 8 & 10,8 & 15 & 20,3 \\
\hline $31---\mid 40$ & 4 & 5,4 & 2 & 2,7 & 25 & 33,8 & 31 & 41,9 \\
\hline | 41 |-- 53 & 4 & 5,4 & - & - & 24 & 32,4 & 28 & 37,8 \\
\hline Total & 12 & 16,2 & 5 & 6,8 & 57 & 77,0 & 74 & 100,0 \\
\hline
\end{tabular}

Através da Tabela 1 pode-se observar que grande parte dos trabalhadores da amostra pertenciam ao sexo feminino $(74,3 \%)$, eram casados $(59,5 \%)$ e tinham idades compreendidas entre 21 e 53 anos.
Em relação a alguns dados antropométricos foi constatado que o peso dos sujeitos oscilou entre $45 \mathrm{Kg}$ e $104 \mathrm{Kg}$ e a altura entre 1,47 metros até 1,84 metros. Através do cálculo do índice de massa corporal (IMC)

**** $\mathrm{Na}$ Argentina o enfermeiro que tem formação de $3^{\circ}$ grau (três anos de graduação) e cursa por mais dois anos a licenciatura é denominado Enfermeiro Universitário (licenciado)

***** Enfermeiro não universitário é aquele que apenas cursou os três anos de graduação

****** Os Auxiliares recebem formação durante 12 meses sem exigência de segundo grau 
com base na classificação recomendada pela INTERNATIONAL LIPID INFORMATION BOREAU (1994) constatou-se que $38(51,4 \%)$ sujeitos apresentavam índices menores de $25 \mathrm{Kg} / \mathrm{m}^{2}$, valores considerados normais; 18 (24,3\%) apresentavam sobrepeso, $6(8,1 \%)$ sujeitos apresentavam obesidade grau I leve e $12(16,2 \%)$ sujeitos foram considerados portadores de obesidade grau II moderada.

Segundo BULHÕES (1986), diante da falta de atenção aos valores antropométricos dos trabalhadores em muitos hospitais, cabe ao trabalhador essa situação passando a ser entendida como esticar, entortar e contundir o corpo às exigências do trabalho.

Os trabalhadores de enfermagem tinham salários diferentes de acordo com a categoria profissional. 62,1\% dos sujeitos recebiam entre US\$ 500 e 1000 (dólares) mensais, $35,2 \%$ entre US\$ 1000 e 1500 (dólares) e apenas 2,7\% tinham salários inferiores a US\$ 500 dólares.

Foi observado que $77 \%$ dos sujeitos não possuíam outro emprego e somente $23 \%$ dos sujeitos trabalhava em um segundo emprego, sendo este também na enfermagem.

A carga horária semanal dos trabalhadores de enfermagem era de 40 horas para $87,8 \%$ dos entrevistados e 44 horas para $12,2 \%$ deles.

Quanto a satisfação do trabalhador para com o trabalho foi constatado que $55(74,3 \%)$ dos sujeitos estavam satisfeitos, $10(13,5 \%)$ insatisfeitos e 9 sujeitos $(12,2 \%)$ estavam satisfeitos pelo tipo de trabalho mas insatisfeitos com as condições de trabalho oferecidas.

Segundo DEJOURS (1992), nenhuma adaptação do trabalho é possível diante das condições de insatisfação.

As condições de saúde constituem-se em um dado importante quando é analisada a adequação da situação de trabalho.

Dentre os entrevistados 44 (59,5\%) trabalhadores referiram não apresentar problemas de saúde, no entanto, $30(40,5 \%)$ trabalhadores referiram problemas. Tais problemas, evidenciados em 38 casos, relacionavam-se a vários sistemas:39\% osteo-musculares, $16 \%$ metabólicos, $13 \%$ neurológicos, $8 \%$ respiratórios, $11 \%$ outros.

$\mathrm{Na}$ opinião de $21(28,4 \%)$ trabalhadores seus problemas de saúde estavam relacionados ao trabalho. Lesões osteo-musculares, tensão emocional e afecções do sistema respiratório foram os problemas de saúde identificados pelos sujeitos como relacionados ao trabalho.
SILVA (1999) descreve que problemas de coluna, lombalgias, varizes, fadiga nervosa, insônia, hipertensão arterial, gastrite, hepatite, infecções fúngicas e de vias aéreas são queixas comuns na história clínica ocupacional de trabalhadores de saúde no setor hospitalar.

Sobre os acidentes de trabalho ocorridos nos últimos doze meses, $18(24,3 \%)$ trabalhadores de enfermagem informaram terem sido acometidos por infortúnios com lesões principalmente por picadas com agulha, cortes, quedas e torções.

\section{Características das atividades de trabalho}

O enfermeiro profissional (universitário ou não) segundo a legislação Argentina, planeja, implementa, executa, dirige e avalia a atenção dos cuidados de enfermagem e tem o auxiliar sob sua responsabilidade. São área de sua competência a assistência, docência, administração e investigação em enfermagem. Quanto ao auxiliar de enfermagem realiza procedimentos básicos de higiene $\mathrm{e}$ alimentação, administra medicamentos, inclusive injetáveis e auxilia o enfermeiro em atividades especiais (ARGENTINA, 1993).

Foi constatado que na instituição estudada as tarefas prescritas se distanciam das tarefas efetivamente realizadas uma vez que todos os sujeitos da amostra referiram prestar os mesmos cuidados de enfermagem aos pacientes independente da categoria profissional, exceção observada apenas para atividades administrativas desempenhadas pelos enfermeiros universitários (licenciados).

Um fator importante para a adaptação entre o trabalho e o homem é a formação profissional (capacitação) e o treinamento.

Segundo SANTOS et al. (1991), as atividades executadas pelos trabalhadores de enfermagem apresentam situações que exigem tomadas de decisões e organização de tarefas originando cargas as quais podem ser reduzidas, através de adequado preparo profissional.

Segundo IIDA (1997) o treinamento constitui uma atividade organizada e programada para melhorar as habilidades de uma pessoa, ou seja para aumentar a velocidade e a qualidade psicomotora dos movimentos necessários para se executar uma tarefa.

Foi observado que $71(95,9 \%)$ sujeitos referiram não ter recebido treinamento ao iniciar seu trabalho no hospital e $3(4,1 \%)$ referiam ter recebido. Grande parte dos sujeitos não consideram a presença de riscos no trabalho. Através da Tabela 2 são apresentadas as opiniões dos trabalhadores a esse respeito. 
Tabela 2 - Número e porcentagem de trabalhadores de Enfermagem de um H.R., segundo a opinião sobre a exposição a cada tipo de riscos ocupacionais. Argentina, 1999

\begin{tabular}{|c|c|c|c|c|c|c|}
\hline \multirow[b]{3}{*}{ TIPOS DE RISCOS } & \multicolumn{6}{|c|}{ EXISTÊNCIA DE RISCOS } \\
\hline & \multicolumn{2}{|c|}{ Sim } & \multicolumn{2}{|c|}{ Não } & \multicolumn{2}{|c|}{ TOTAL } \\
\hline & $n^{\circ}$ & $\%$ & $n^{\circ}$ & $\%$ & $n^{\circ}$ & $\%$ \\
\hline Biológico & 21 & 28,4 & 53 & 71,6 & 74 & 100,0 \\
\hline Físico & 11 & 14,9 & 63 & 85,1 & 74 & 100,0 \\
\hline Químico & 5 & 6,8 & 69 & 93,2 & 74 & 100,0 \\
\hline Outros & 10 & 13,5 & 64 & 86,5 & 74 & 100,0 \\
\hline
\end{tabular}

Dentre os riscos ocupacionais detectados pelos sujeitos os mais freqüentes estavam relacionados a agentes biológicos.

Através desses resultados pode-se observar uma lacuna no conhecimento desses profissionais por desconhecerem o próprio ambiente de trabalho.

\section{Características do ambiente de trabalho}

Através da Tabela 3 são apresentados as opiniões emitidas pelos trabalhadores de enfermagem sobre as condições do ambiente de trabalho.

Tabela 3 - Número e porcentagem de trabalhadores de enfermagem do H.R., segundo a opinião acerca das condições do ambiente de trabalho. Argentina, 1999

\begin{tabular}{|c|c|c|c|c|c|c|}
\hline \multirow{2}{*}{$\begin{array}{c}\text { CONDIÇÕES DO } \\
\text { AMBIENTEDE } \\
\text { TRABALHO }\end{array}$} & \multicolumn{2}{|c|}{ ADEQUADO } & \multicolumn{2}{|c|}{ INADEQUADO } & \multicolumn{2}{|c|}{ TOTAL } \\
\hline & $n^{0}$ & $\%$ & $n^{0}$ & $\%$ & $n^{\circ}$ & $\%$ \\
\hline $\begin{array}{l}\text { Disposição de } \\
\text { equipamentos }\end{array}$ & 59 & 79,7 & 15 & 20,3 & 74 & 100,0 \\
\hline Ihmimação & 54 & 73,0 & 20 & 27,0 & 74 & 100,0 \\
\hline Espaçodo ambiente & 41 & 55 , & & & 74 & 100,0 \\
\hline Ruídos & 31 & 41,9 & 43 & 58 & 74 & 100,0 \\
\hline Termperatura & 29 & 392 & 45 & 60,8 & 74 & 100,0 \\
\hline Ditância perconida & 29 & 392 & 45 & 60,8 & 74 & 100,0 \\
\hline
\end{tabular}

A iluminação foi considerada adequada por 54 $(73 \%)$ trabalhadores e inadequada por $20(27 \%)$ sujeitos.

Segundo BLANPAIN \& ESTRYN-BEHAR (1990) as diferentes unidades que compõem o hospital devem ter iluminação de acordo com a atividade realizada. O trabalho realizado no posto de enfermagem necessita de iluminação de 500 LUX em outras unidades do hospital deve ser variável entre 200 e 300 LUX, exceto centro cirúrgico.

Embora não tenha sido aferido o iluminação nos postos de trabalho, observou-se que poucos sujeitos estavam atentos às questões de riscos ocupacionais provenientes de ambientes inadequadamente iluminados.

Em relação à temperatura do ambiente de trabalho, $45(60,8 \%)$ sujeitos consideraram-na inadequados e $29(39,2 \%)$ adequada. Segundo os entrevistados, a temperatura é controlada pelo pessoal da manutenção, o sistema de calefação central utilizado não mantém a temperatura estável e regular, alternando momentos de temperaturas extremas $\left(40^{\circ} \mathrm{C}\right)$ o que na opinião de $60,8 \%$ da amostra estudada, provoca desconforto térmico, uma vez que a temperatura extrema é baixa, cerca de $-10^{\circ} \mathrm{C}$.

$\mathrm{Na}$ legislação argentina, a lei $\mathrm{n}^{\circ} 19.507$ que trata da "Seguridad e higiene en el trabajo", estabelece limites para a carga térmica do ambiente de trabalho, segundo o tipo de trabalho. A referida legislação estabelece para trabalhos leves, a temperatura de $30^{\circ} \mathrm{C}$ e trabalhos moderados de $26,7^{\circ} \mathrm{C}$ (ARGENTINA, 1998).

O desconforto térmico acarreta prejuízos á saúde dos trabalhadores e as temperaturas elevadas não são adequadas ao ambiente hospitalar devido a proliferação de microorganismos acelerada pelo calor, elevando o risco de infecções entre pacientes e trabalhadores. Temperaturas muito baixas também podem prejudicar a saúde dos trabalhadores levando a ocorrência de afecções respiratórias, reumáticas, neurológicas, otites, conjuntivites dentre outros (VAQUERO PUERTA \& CENÃ VALLEJO ,1996).

Segundo ODDONE et al. (1986), o excesso de calor no ambiente ocasiona diminuição da capacidade de concentração e o aumento da fadiga.

Em relação ao ruído foi constatado que 31 $(41,9 \%)$ trabalhadores de enfermagem consideraram os níveis de ruído no ambiente de trabalho adequado e 43 $(58,1 \%)$ inadequado por ocasionar desconforto acústico devido ao barulho emitido de fontes tais como telefone, aparelho de calefação, campainha e alarmes, equipamento usado para limpeza dentre outros.

O nível de ruído no hospital segundo MARZIALE (1995) deve estar estabelecido entre 35 e $45 \mathrm{~dB}$ (A) para promover conforto acústico aos pacientes (que precisam recuperar-se) e aos trabalhadores (que precisam de segurança no trabalho).

Segundo a legislação argentina nenhum trabalhador poderá estar exposto a ruídos contínuo e constante superior a $90 \mathrm{~dB}$ (A) em jornada de 8 horas. Deverá ser providenciada proteção auditiva para trabalhadores com exposição a ruídos maior ou igual a 115 dB (A) ARGENTINA (1998).

O espaço do ambiente de trabalho, a disposição de equipamentos foram considerados adequados por mais da metade dos trabalhadores, no entanto as distâncias percorridas durante a jornada de trabalho foram consideradas inadequadas por $60,8 \%$ dos trabalhadores.

A maior parte dos trabalhadores de enfermagem que emitiram opiniões sobre os problemas da situação de trabalho apontaram aspectos relacionados a organização do trabalho e ao relacionamento interpessoal intra e inter equipes profissionais. 


\section{CONSIDERAÇÕES FINAIS}

Através dos resultados obtidos, foi possível diagnosticar a seguinte situação de trabalho do pessoal de enfermagem:

- Os trabalhadores de enfermagem predominantemente pertenciam ao sexo feminino $(74,3 \%)$, eram casados (59,5\%), possuíam idades entre 30 e 53 anos (79,7\%), tinham peso entre 45 a $104 \mathrm{Kg}$ e altura entre $1,47 \mathrm{~m} \mathrm{e}$ $1,84 \mathrm{~m}$ sendo observado que $48,6 \%$ dos sujeitos apresentaram pesos acima dos valores considerados normais quando calculado a IMC. A maioria dos trabalhadores tinha carga horária semanal de 40 horas e não possuíam um segundo emprego. Grande parte $(74,3 \%)$ dos sujeitos estavam satisfeitos com o trabalho e $40,5 \%$ trabalhadores referiram problemas de saúde, os quais na opinião de $28,4 \%$ dos sujeitos estavam relacionados as condições de trabalho. Apenas $24,3 \%$ dos trabalhadores referiram terem sido acometidos por acidentes de trabalho nos últimos doze meses provocando-lhes lesões por picadas, cortes, quedas e torções.

- Em relação as atividades de trabalho embora as atividades formais encontraram-se legalmente estabelecidas efetivamente foi observada que os enfermeiros e os auxiliares de enfermagem executavam as mesmas tarefas. A maioria dos sujeitos considerou a demanda de esforço físico e mental significativo para a execução das tarefas de enfermagem. A falta de treinamento foi apontada como um problema a ser resolvido. Assim como a falta de informações dos trabalhadores sobre os agentes de riscos peculiares ao ambiente de trabalho.

- Na opinião dos trabalhadores de enfermagem constituíam-se problemas no ambiente de trabalho principalmente a falta de organização do trabalho e a dificuldade de comunicação e relacionamento inter e intra equipes de enfermagem e médica. Os trabalhadores demonstraram falta de conhecimentos sobre os riscos ocupacionais do ambiente de trabalho.

Apesar do estudo ora apresentado ter buscado apenas diagnosticar a situação de trabalho, algumas sugestões, com base na ergonomia de correção, são apresentadas: motivar a participação conjunta da gerência, dos trabalhadores e de especialistas (assessores) com vistas a discutir sobre a organização do trabalho; utilizar estratégias para melhorar o relacionamento e a comunicação intra e inter equipes; oferecer treinamento e programas de reciclagem; orientar os trabalhadores quanto aos fatores de risco do ambiente hospitalar e das atividades executadas; conscientizar e estimular os trabalhadores ao uso de Equipamento de Proteção Individual; reorganizar a distribuição de atividades de trabalho de acordo com cada categoria profissional; adequar ergonomicamente os espaços físicos; adequar os equipamentos e mobiliários, de acordo com os padrões antropométricos dos trabalhadores; adequar a temperatura, o nível de ruído e a iluminação dos postos de trabalho; incentivar a realização de pesquisas e aplicação dos resultados das mesmas a fim de adequar a prática profissional e a situação de trabalho.

\section{THE WORK SITUATION OF THE NURSING PERSONNEL FROM AN ARGENTINE HOSPITAL: A STUDY BASED ON ERGONOMICS}

The present study aimed at characterizing the work situation of the nursing personnel from an Argentine Hospital through the identification of the characteristics of the workers, the executed activities and the work environment, using the methodological fundamentals of ergonomics. In order to diagnose the work situation, the author performed 74 interviews with the workers, 17 nurses and 57 nursing auxiliaries. Findings showed that the work situation in the studied presents problems related to the work organization, tasks division, lack of a policy directed to improve professional capacities and safety at work, promotion of worker's health and adequacy of the materials, equipments. The authors presented suggestions based on ergonomics in order to minimize or to eliminate the detected problems.

\section{LA SITUACIÓN DE TRABAJO DEL PERSONAL DE ENFERMERÍA EN EL CONTEXTO DE UN HOSPITAL ARGENTINO: UN ESTUDIO SOBRE LA ÓPTICA DE LA ERGONOMÍA}

Con el objetivo de caracterizar la situación de trabajo del personal de enfermería en un hospital argentino a través de la identificación de las características de los trabajadores, de las actividades ejecutadas y del ambiente laboral, se utilizó como presupuesto metodológico la Ergonomía. Buscando el diagnóstico de la situación de trabajo fueron realizadas entrevistas con 74 trabajadores, siendo 17 enfermeros y 57 auxiliares de enfermería. Por medio de los resultados obtenidos, se constato que la situación de trabajo estudiada presente problemas relacionados con la organización del trabajo, división de tareas, falta de 
política orientada al mejoramiento de la capacitación profesional y la seguridad en el trabajo, promoción a la salud de los trabajadores, adecuación de materiales y equipos. Con base en la Ergonomía de corrección se presentan sugerencias.

TÉRMINOS CLAVES: enfermería, trabajo, ergonomía

\section{REFERÊNCIA BIBLIOGRÁFICA}

01. AGUDELO, M.C.C. El trabajo en enfermería. In: MACHADO, M.H. Profissões de saúde: uma abordagem sociológica. Rio de Janeiro: FIOCRUZ, 1995.

02. ARGENTINA Ministerio de Salud y Acción Social. Ley 24.004. Normas del ejercicio de enfermería. Bs. As. Boletin oficial $\mathbf{N}^{\mathbf{0}} \mathbf{2 7 . 7 8 7}, 1^{\circ}$ sección. Diciembre 1993. 22p.

03. ARGENTINA. Seguridad e higiene en el trabajo.Córdoba (Argentina): Editorial la Canadá, 1998. 135p.

04. BLANPAIN, G.; ESTRYN-BEHAR, M. Mensures d'ambiance phisique dans dix services hospitaliers. Perfomances, London, n.45, p.1833, jan./fev. 1990.

05. BRASIL Ministério de Saúde. Secretaria de Assistência à Saúde. Conselho Nacional de Saúde. Diretrizes e normas regulamentadoras de pesquisa envolvendo a seres humanos. Brasília: Ministério de Saúde, 1997. 20 p.

06. BULHÕES, J. Enfermagem do trabalho. Rio de Janeiro: Ed. IDEAS, 1986. v. 2.

07. DEJOURS, C. A loucura do trabalho. Estudo de psicopatologia do trabalho. Trad. Ana Isabel Paraguay e Lúcia Leal Ferreira. São Paulo: Cortez, 1992.

08. FISCHER, F.M.; PARAGUAY, A.I.B.B. A ergonomia como instrumento de pesquisa e melhoria das condições de vida e trabalho. In: FISCHER, F.M. et al. Tópicos de saúde do trabalhador. São Paulo: HUCITEC, 1989. p. 19-72.

09. IIDA, I. Ergonomia. Projeto e produção. São Paulo: Edgard Blücher, 1997.
10. INTERNATIONAL LIPID INFORMATION BUREAU (ILIB). Latino América Recomendaciones de ILIB para el diagnóstico de las dislipidemias en Latinoamérica Cardiovasc. Risk Factors, v. 3, n. 1, p. 10-27, 1994. Suplemento 1.

11. MARZIALE, M.H.P. Condições ergonômicas da situação de trabalho, do pessoal de enfermagem, em uma unidade de internação hospitalar. Ribeirão Preto, 1995. 163p. Tese (Doutorado) - Escola de Enfermagem de Ribeirão Preto, Universidade de São Paulo.

12. ODDONE, I. et al. Ambiente de trabalho: luta de trabalhadores pela saúde. São Paulo: HUCITEC, 1986. 133p.

13. SANTOS, N. et al. Saúde e trabalho dos operários da saúde: um diagnóstico da ergonomia. In: SEMINÁRIO BRASILEIRO DE ERGONOMIA, 5, São Paulo, 1991. Anais. São Paulo, 1991. p. 199-200.

14. SANZ-GALLÉN, P. et al. Introducción a la salud laboral. In: SANZ-GALLÉN, P.; IZQUIERDO, J.; PRAT MARÍN, A. Manual de salud laboral. Barcelona: Springer-Verlag Ibérica, 1995. p. 17.

15. SILVA, D.M.P.P. O adoecer dos trabalhadores de enfermagem: um estudo dos problemas de saúde responsáveis pelo absenteísmo-doença em um hospital universitário. Ribeirão Preto, 1999. 139p. Dissertação (Mestrado) - Escola de Enfermagem de Ribeirão Preto, Universidade de São Paulo.

16. VAQUERO PUERTA, J.L.; CEÑA VALLEJO, R. Prevensión de riesgos laborales: seguridad, higiene y ergonomía. Madrid: Ed. PIRAMIDES, 1996. 